\title{
Participar en la gestión local: los actores urbanos y el control fiscal cívico en Bogotá
}

\author{
THIERRY LULLE*
}

\begin{abstract}
During 1980's a processes of decentralization and democratization have began in Colombia. Since that period the consensus as a style of social participation was consolidated, particularly in the context of planning and management of urban development. Despite participation experiences multiplied, they were not always successful. This article offers an analysis of these new processes of social participation through a case study on civic control carried out in Bogota between 1997 and 1999, which main goal was to involve local communities on monitoring the implementation of the city's public budget in local development projects. With this case study this article argues that social participation is not only a mean of establishing rational and equitable procedures of local management, but also a mean of reorganizing the traditional system of the sociopolitics of urban actors.
\end{abstract}

Keywords: Bogota, civic control carried out, management of local development, social participation, urban actors.

\section{Resumen}

Durante la década de 1980 se iniciaron en Colombia los procesos de descentralización y democratización. Desde entonces se afirma un discurso consensual en torno a la participación ciudadana, en especial en materia de planeación y gestión del desarrollo urbano. Sin embargo, si bien se multiplicaron experiencias en este sentido, no siempre fueron exitosas. Este artículo ofrece un análisis de estos nuevos procesos a través de la presentación y evaluación de una experiencia de control fiscal cívico que se llevó a cabo en Bogotá entre 1997 y 1999, y cuyo objetivo era propiciar la participación de los habitantes en el control de la cabal ejecución de los fondos públicos invertidos en proyectos de desarrollo local. Se trata de mostrar cómo el discurso de la participación puede servir no tanto para lograr una gestión más racional y equitativa, sino para la reorganización del sistema tradicional de actores socio-políticos urbanos.

Palabras clave: Bogotá, control fiscal cívico, gestión local, participación ciudadana, actores urbanos.

\footnotetext{
*Universidad Externado de Colombia. Correo-e: th.lulle@elsitio.net.co
} 


\section{Introducción}

Hoy en día la participación de los ciudadanos en la gestión pública es a menudo promovida particularmente en el campo del desarrollo urbano, el cual involucra numerosos actores: los habitantes mismos, pero también la administración pública, los políticos, el sector privado, entre otros. Se trata de romper con formas de poder tradicionales poco democráticas y equitativas, tales como el clientelismo y el autoritarismo; también con una planeación desconectada de las necesidades reales de la población, sobre todo la de pocos recursos. Se pretende que los ciudadanos se empoderen, facilitándoles el acceso a los dispositivos institucionales y que se responsabilicen más en la gestión de sus espacios de vida. Sin embargo, la amplia divulgación y apropiación del discurso de la participación no viene acompañada de una aclaración suficiente de su significado y menos todavía de una aplicación fluida al igual que sucede con otras nociones complementarias como comunidad, ciudadanía, sociedad civil. Si bien en las últimas décadas, tanto en los países del norte como del sur (CNUAH, 1996) se han llevado a cabo experiencias exitosas y muy difundidas (por ejemplo el presupuesto participativo de Porto Alegre en Brasil) muchas otras, al haber sido difíciles o incluso al haber fracasado, señalan lo arduo, complejo e incluso ambiguo de la concreción de la participación ciudadana. Por lo tanto, estamos frente a una situación paradójica que invita al análisis.

Cabe recordar que el carácter unívoco del discurso de la participación apareció recientemente. En efecto, mientras algunos de los actores que hoy la promueven eran caracterizados hace tres décadas como 'autoritarios', en ese mismo periodo otros la reivindicaban en nombre de los principios de la 'autogestión' en el centro de luchas urbanas, a veces duramente reprimidas. Estos conflictos cuestionaban no solamente los poderes económicos y políticos dominantes, poco preocupados por las necesidades de las nuevas masas urbanas, sino también el 'poder de los sabios' (los planificadores) frente a los 'derechos de los profanos' (los habitantes).

Si bien la idea de la participación en el proceso de la construcción de los espacios habitados se expresó desde los inicios de la teorización de la arquitectura en el Renacimiento occidental, ésta fue a la postre muy poco desarrollada en la teoría y la práctica. El tratadista italiano Alberti ha insistido en la necesidad de un diálogo entre el arquitecto y el cliente -o los futuros usuarios del pro- 
yecto en diseño- y ha propuesto una metodología para clasificar estos mismos usuarios con el fin de responder mejor a sus necesidades y deseos. Si bien el tema no estuvo ausente en las teorías subsecuentes, hubo que esperar hasta la década de los años 70 para que el procedimiento de la participación fuese objeto de reflexión y experiencia sistematizadas. En otros términos, podemos decir que incluso cuando 'los sabios' cuestionaron su propio poder y trataron de plantear conceptos y métodos para abrir su práctica a sus interlocutores 'los profanos', la tentativa tuvo un impacto limitado: la participación en sí tendría un carácter bastante 'subversivo'.

Vale la pena recordar que en las evaluaciones de las experiencias sobre participación destacan varias dificultades de fondo (Blanc, 1995; Lorcerie, 1995; Atkinson, 1998), que se resumen en el hecho de que no siempre la población se apropia de estos nuevos espacios. Con frecuencia se invoca la falta de cultura o educación política de las masas, pero también pueden ser identificadas otras causas. Es claro que si la iniciativa proviene no de 'abajo' sino de 'arriba', las reglas del juego cambian radicalmente. También se ha mostrado cómo el enfoque participativo, ligado a un moderno proceso de racionalización política, viene a cuestionar una organización socio-política más tradicional (Vidal, 1998). Otra causa es el desfase, a veces demasiado grande, entre los intereses de cada actor involucrado en la gestión local. Este desfase puede acentuarse todavía más cuando se le sobrepone una gran diversidad de lenguajes y, por tanto, de interpretaciones del procedimiento mismo de la participación. Finalmente, hay que tener en cuenta la temporalidad variable de la motivación de los habitantes: ésta disminuye tan pronto se satisfacen las necesidades básicas.

A pesar de estas dificultades el discurso de la participación se ha generalizado e, indudablemente, ello se debe a las orientaciones emanadas de las organizaciones internacionales desde finales de la década de los años 80, quienes dicen valorar la descentralización, la democratización, la governanza, la concertación. Pero, al mismo tiempo, ejercen presiones sobre los gobiernos nacionales para que se apliquen drásticas medidas en materia económica, especialmente en los países del sur. Esta ambigüedad se reproduce en el ámbito nacional cuando, como consecuencia de las presiones internacionales, los gobiernos tienen que neutralizar situaciones sociales a veces explosivas. Con este fin promueven el discurso 'políticamente correcto' de la democratización de las ins- 
tituciones públicas y favorecen ciertos dispositivos locales 'incluyentes', aunque de manera superficial.

Para analizar mejor esta situación, y aprovechando la coyuntura -quizás más reveladora- de desfase agudo entre discursos y prácticas, en este texto se propone una hipótesis que muestra la naturaleza de las dinámicas propiamente locales: La participación es percibida por los actores organizados como una amenaza, pues puede someter a examen sus prácticas marcadas por el clientelismo, pero como estos actores (ligados por ser partícipes de un sistema tradicional, basado en la representación política y bajo un régimen clientelar) no pueden ir en contra de la participación, prefieren neutralizarla y más bien reutilizarla como un espacio para reequilibrar sus interacciones.

Planteamos esta hipótesis con base en la sistematización de una experiencia de participación llevada a cabo entre 1997 y 1999 en Bogotá, Colombia. Se trató de un programa de control fiscal cívico puesto en marcha por la Contraloría de Bogotá que preconizaba el ejercicio del control de la ejecución en el ámbito local de las inversiones públicas por los ciudadanos beneficiarios de ellas. Por lo tanto, la participación era promovida desde arriba, pero convocó al conjunto de los actores involucrados en el desarrollo local.

En Colombia, como en muchos otros países de América del sur, a finales de la década de los años 80 se iniciaron fuertes procesos de descentralización y democratización. Paralelamente a la introducción de la participación a través de leyes y de numerosas experiencias (Velásquez y González, 2003), se realizaron reformas del sistema de representación política, aunque no libres de tensiones. En efecto, el tejido socio-político de la ciudad estaba marcado por una organización jerárquica entre los ámbitos municipal y barrial, a los cuales dichas reformas podían afectar directamente. Al mismo tiempo, como en muchas otras partes, un sector del sistema político tradicional de partidos parecía entrar en crisis de confianza con sus electores (creciente abstencionismo, emergencia de figuras independientes), y otro sector, las organizaciones barriales -muy fuertes al momento de reivindicar la satisfacción de necesidades básicas- que podían ser menos valoradas una vez logradas sus metas. A su vez, los contenidos y las formas de la planeación y gestión del desarrollo urbano fueron ajustados en el sentido de una mayor racionalización y democratización. Los efectos de estos cambios se advirtieron especialmente en Bogotá, pues entró en una nueva fase de recuperación de sus espa- 
cios públicos bastante afirmada. Estas dinámicas específicas llevan a plantear interrogantes más generales:

i. ¿La aparente voluntad de favorecer la democracia participativa está ligada a la supuesta crisis de la democracia representativa?;

ii. ¿Será que la participación siempre está aceptada por los actores locales?, ¿no puede afectar y cuestionar acuerdos y reglas establecidas y aceptadas por ellos desde hace mucho tiempo?;

iii. ¿En qué medida la participación favoreció un mejoramiento de la planeación y gestión del desarrollo local?

A continuación se presenta un contexto general y un análisis del desarrollo de las democracias representativa y participativa en Bogotá -tal como se dio a través de la reorganización políticoadministrativa de las localidades-, posteriormente se presentará y evaluará el programa de control fiscal cívico, resaltando las formas de su apropiación por parte de los diferentes actores, así como los juegos entre discursos y prácticas y el provecho obtenido por cada actor. Esto nos permitirá plantear una hipótesis sobre la paradoja en la que se encuentra el actual desarrollo de la participación.

\section{Contextos político, económico y social del desarrollo urbano de Bogotá}

Colombia es un país caracterizado por fuertes diferencias regionales. Algunas regiones se desarrollaron económicamente desde el siglo XIX a través de la industrialización, agro-industrialización y comercialización. La urbanización se acentuó a partir de mediados del siglo pasado, alcanzando en 1993 una tasa de 69.1\%. Este proceso se apoyó en una red urbana 'cuadricefálica' (Bogotá y las tres grandes ciudades: Medellín, Cali y Barranquilla) que quedó equilibrada durante un largo tiempo; sin embargo, a partir de la década de 1970 en la capital empezó a predominar una concentración de actividades financieras y de servicios ligadas a la mundialización de la economía (Gouëset, 1998). Así, si bien el desarrollo económico contribuyó al bienestar de algunos sectores de la población, también se acompañó de fuertes desigualdades y exclusiones, tanto en el campo como en las ciudades. 
Esta evolución fue marcada por la naturaleza del sistema político: unos poderes regionales independientes, fuertemente jerarquizados y marcados por estructuras sociales tales como el paternalismo y el clientelismo, que debilitaron al Estado nacional y se opusieron profundamente a la confrontación social pública y directa. La violencia pudo ser utilizada al servicio de estas formas de dominación. Se habló de una situación paradójica entre un orden democrático (casi nunca Colombia conoció la dictadura) y una violencia variablemente intensa y subterránea (Pécaut, 1987). En la década de 1950 el tradicional conflicto entre los dos grandes partidos políticos -el liberal y el conservador-se agudizó fuertemente ("la Violencia") a costa de más de doscientos cincuenta mil muertos. Durante la siguiente década hubo reconciliación ("el Frente Nacional”), poniendo fuera del nuevo orden a corrientes más extremistas, que optaron por la clandestinidad y la lucha armada. Mientras tanto, el narcotráfico se desarrolló e infiltró progresivamente no sólo en los partidos políticos oficiales, sino también en los grupos clandestinos (guerrillas y paramilitares). Desde la década de 1980 se entablaron periódicamente negociaciones entre el Estado y las guerrillas, pero sin éxito, salvo con una de ellas: el Movimientos 19 de abril (м19) en 1990.

Durante la década de 1990, mientras los conflictos se volvían cada vez más complejos, se desarrolló un proceso de democratización política apoyada en los principios de justicia y participación invocados en la nueva Constitución de 1991. Esta nueva carta política pretende responder a necesidades específicamente locales de reconciliación (la Constituyente incluyó al M19, así como a las minorías étnicas), pero se inscribe también en un contexto latinoamericano marcado por la descentralización, en el marco de la cual se inician varias reformas: la transferencia de competencias y recursos de la nación a las entidades locales, una reorganización político-administrativa, la puesta en marcha de procesos de planificación y gestión locales, la preconización de formas de participación de la ciudadanía en la toma de decisiones y en el control de la adecuada ejecución de éstas. No obstante, si bien dichas transformaciones tendieron a dar una nueva legitimidad a las instituciones públicas, facilitaron también la apertura económica a principios de la década de los años 90. A pesar de sus graves problemas, el país mantuvo hasta hace poco una economía internacionalmente considerada como equilibrada, poco afectada por la crisis internacional de 1985 , con una inflación conside- 
rablemente inferior a la de la mayoría de los demás países de la región. Según la opinión de algunos analistas, el narcotráfico explicaría parcialmente este equilibrio. Sin embargo, a finales de la década de 1990 surge una crisis económica de una amplitud hasta ahora desconocida en este país.

La democratización política se basó en reformas del dispositivo de representación (especialmente con la elección popular de los alcaldes y gobernadores a partir de 1988) y en la creación de instancias y procedimientos para permitir la participación de los ciudadanos en la gestión pública. Estos cambios se llevaron a cabo mientras el tejido socio-político trató de reorganizarse. Por un lado, la crisis de los partidos políticos tradicionales favoreció la emergencia de nuevas figuras llamadas independientes, pero éstas difícilmente se fortalecieron; mientras las redes clientelistas de los partidos siguen activas. Por otro lado, los movimientos sociales sufrieron cierta degradación en las décadas de 1960 y 1970. Ello estuvo motivado, en gran medida, por las reivindicaciones de regularización de los barrios ilegales y auto-construidos, y de su equipamiento de servicios domiciliarios, todo lo cual había sido canalizado a través de las Juntas de Acción Comunal (JAC). Es decir, organizaciones barriales creadas por ley en 1958 mismas que se encuentran actualmente bastante incrustadas y a menudo ligadas a redes clientelares.

Bogotá, con una población en 1993 de cerca de seis millones de habitantes, reflejó las dinámicas del conjunto del país, aunque con ciertas especificidades. El Distrito ${ }^{1}$ se expandió rápidamente en las décadas de 1950 y 1960, lo cual se explica por una tasa muy alta de crecimiento demográfico, a la cual contribuyó la llegada masiva de migrantes. Esta expansión se produjo a menudo de forma ilegal, pues la insuficiente aplicación por parte de los poderes públicos de sus políticas y planes amplió el desfase entre una demanda vertiginosamente creciente y una oferta insuficiente: estas dinámicas acentuaron el carácter segregativo de la estructura socio-espacial de la ciudad. A partir de las décadas de 1970 y 1980, los comportamientos demográficos cambiaron: disminución de la natalidad y la mortalidad, reducción y diversificación de la migración. Sin embargo, la tasa absoluta de crecimiento siguió alta, de tal forma que la expansión se concretó con la incorporación de los municipios vecinos y la conformación de un área metropolitana (aunque todavía no formalizada); al mismo

${ }^{1}$ De ahora en adelante llamaremos distrito al municipio de Bogotá. 
tiempo, las zonas ya construidas dentro de los límites del distrito se densificaron. En este contexto, el laxismo de los poderes públicos agravó el ya caótico funcionamiento de la ciudad (congestión del tránsito, inseguridad, degradación de los espacios públicos).

A partir de 1990, y gracias a las nuevas herramientas de democratización y descentralización, la gestión de la ciudad estuvo mejor controlada. Los alcaldes, elegidos desde 1988 por sufragio universal, adoptaron nuevas formas de gestión. Jaime Castro (19921994) intervino en el campo político-administrativo y financiero; Antanas Mockus (1995-1997) desarrolló un discurso sobre la cultura ciudadana; Enrique Peñalosa (1998-2000) realizó grandes obras de recuperación de los espacios públicos, y Mockus (reelegido en 2001) fortaleció estos procesos. Sin embargo, esta indudable reorganización se encuentra a veces limitada por el peso de lógicas clientelistas tradicionales que ligan a los concejales del Distrito con su electorado (el sector económico, los agentes de la administración pública u organizaciones de habitantes). Por otra parte, desde finales de la década pasada, dicha organización está puesta a prueba por una problemática social difícil, causada por la reciente crisis económica y por la llegada masiva de los llamados "desplazados por la violencia”, es decir personas expulsadas por los conflictos armados en las zonas rurales.

\section{La innegable pero difícil apertura de espacios democráticos locales}

En el transcurso de la década de 1980, pero sobre todo a partir de la nueva Constitución de 1991 y la abundante jurisdicción derivada de ella, numerosas reformas se están iniciando tanto en favor de la representación política de los ciudadanos como de su participación. Nos centramos aquí en las reformas impulsadas en el ámbito municipal. ${ }^{2}$

\subsection{La representación política}

La situación en Bogotá es la siguiente (véase el cuadro 1):

${ }^{2}$ La organización político-administrativa de Colombia tiene cuatro niveles: el nacional (presidente de la República y Congreso), el departamental (gobernador y consejo), el municipal (alcalde y consejo), el local (el alcalde local y la Junta Administradora Local, JAL). 


\section{Cuadro 1}

Sistema de representación política de la población en Bogotá

\begin{tabular}{|c|l|l|}
\hline Nivel territorial* & \multicolumn{1}{|c|}{ Elegidos } & \multicolumn{1}{c|}{ Modalidades } \\
\hline \multirow{2}{*}{ Distrito } & Alcalde mayor & Sufragio universal no obligatorio \\
\cline { 2 - 3 } & Consejo & Sufragio universal no obligatorio \\
\hline \multirow{2}{*}{ Localidad } & Alcalde menor & $\begin{array}{l}\text { Escogido por alcalde mayor en una terna } \\
\text { propuesta por la JAL }\end{array}$ \\
\hline \multirow{2}{*}{ Barrio } & JAL & Sufragio universal no obligatorio \\
\cline { 2 - 3 } & JAC & Elegida por afiliados a la JAC \\
\hline
\end{tabular}

Fuente: elaboración propia

* No se menciona el nivel metropolitano por no tener representación política propia.

- En 1988, la introducción de la elección popular (no obligatoria) del alcalde del distrito; ${ }^{3}$

- Se sigue eligiendo popularmente a los miembros del consejo distrital, quienes discuten y adoptan propuestas que proceden tanto de ellos mismos como del alcalde distrital;

- Otra novedad es la elección popular (no obligatoria) a partir de 1993 de los miembros de las Juntas Administradoras Locales (JAL), llamados ediles. Las JAL consisten en una clase de consejo ubicado en el ámbito de las localidades, las cuales son unidades político-administrativas intermedias entre el distrito y el barrio. ${ }^{4}$ Estas unidades existían ya anteriormente con otro nombre; no cambia mucho su territorio, pero sí sus competencias ahora más determinantes. Las elecciones del alcalde distrital, de los concejales y de los ediles se efectúan el mismo día, cada tres años (un quinto periodo va a empezar a principios de 2004 para las JaL);

- La localidad tiene también un alcalde, el alcalde local, pero éste no es elegido popularmente, sino nombrado por el alcalde del distrito después de haber sido escogido por él dentro de una terna propuesta por la JAL; tiene recursos reducidos y su gestión es bastante controlada por la JAL.

\footnotetext{
${ }^{3}$ Los secretarios y gerentes de departamentos y entidades de la administración distrital son nombrados por el alcalde distrital.

${ }^{4}$ De ahora en adelante usaremos los términos localidad y local para referirnos a esta escala intermedia entre el distrito y el barrio. El territorio del distrito de Bogotá está dividido en 20 localidades, y cada una de ellas en centenares de barrios. Se trata de una división político-administrativa.
} 
- En el ámbito barrial no hay cambio: siguen las Juntas de Acción Comunal (JAC), las cuales son elegidas voluntariamente por los habitantes del barrio. No intervienen sino a este nivel.

\subsection{La participación ciudadana}

Las instancias de participación son múltiples, y aquí mencionaremos sólo las que tienen que ver directamente con los procesos de planeación y gestión del desarrollo urbano y no con otros sectores como la salud, la educación, entre otros.

- En el ámbito municipal se crearon a lo largo de la década pasada los consejos de planeación, las veedurías ciudadanas, los comités de desarrollo y control social de los servicios públicos, los consejos municipales ambientales, los consejos consultivos de ordenamiento, entre otros;

- En el ámbito local, las Jal y los consejos locales de planeación.

Los procedimientos a través de los cuales se puede desarrollar la participación son también varios. Es posible identificar tres etapas: la planeación, la ejecución de los planes y el control de esta misma ejecución. Es principalmente en la primera y tercera etapas que se puede ejercer la participación. Bogotá dispone de tres tipos de planes y para cada uno de ellos se crearon formas de participación específicas:

- Un Plan de Ordenamiento Territorial (РОт), cuya vigencia es de nueve años (el primero fue adoptado en 2000), su objeto es la organización físico-espacial del desarrollo futuro del distrito. Se creó en el ámbito distrital un Consejo Territorial de Planeación (СTP) conformado por 40 miembros, la mitad de ellos son ediles, uno por cada localidad, y la otra mitad son representantes de los diferentes gremios económicos y organizaciones sociales de la ciudad. También el Departamento Administrativo de Planeación Distrital (DAPD) -entidad de la administración distrital encargada de la planeación físico-espacial del distrito- realizó talleres de información en las localida- 
des. En cada localidad existe un Consejo Local de Planeación (CLP), reproducción del CTP en este ámbito.

- Un plan de desarrollo propio de cada alcalde, cuya vigencia es de tres años. Este plan con sus objetivos y metas determina la inversión de los fondos públicos del distrito. Se adopta en el consejo distrital, pero resulta también de un proceso de concertación (con este fin fueron creados los llamados 'encuentros ciudadanos') con las localidades que elaboran en paralelo su propio plan: el Plan de Desarrollo Local (PDL), cuya vigencia es también de tres años. Debe haber complementariedad entre el plan distrital y los 20 PDL.

- El PDL se elabora con base en un trabajo de identificación de necesidades y de definición y priorización de proyectos en el ámbito barrial (etapa a la cual contribuyen directamente las JAC), y enseguida en el ámbito local. Las JAL y los encuentros ciudadanos constituyen espacios de debates, pero los PDL son adoptados en las primeras.

En las etapas de gestión (ejecución y control), para el ámbito distrital existen instituciones públicas (contraloría, personería, defensoría), cuyas misiones consisten directamente en la función de control. Hay que precisar que, en el caso de la primera de ellas, el contralor es elegido por el consejo, lo que puede sesgar su imparcialidad. Pero en el ámbito local es nuevamente la JAL la que tiene que asumir esta función o al menos facilitarla. En todos los ámbitos la ley permite la conformación de veedurías ciudadanas, finalmente algunas instancias administrativas pudieron promover programas de participación, como es el caso de la contraloría con respecto del control fiscal cívico.

Estos cambios de diferente naturaleza generan una multiplicación de experiencias en el país a todas las escalas territoriales (de la nación al barrio, pasando por el departamento, el municipio y la localidad) de amplitud, eficacia y divulgación muy variables (por ejemplo, unos cabildos en comunidades indígenas, el sistema regional de planificación participativa en la región del Magdalena medio, la cual es víctima desde hace varios años de conflictos armados muy violentos, la planeación participativa en áreas metropolitanas, municipios, localidades, el presupuesto participativo de Pasto, ente muchos otros). ${ }^{5}$ Aunque lentamente, estas expe-

${ }^{5}$ Véase Velásquez y González, 2003. 
riencias favorecen la emergencia de una nueva cultura de la planificación y gestión en los actores urbanos, ya que favorecen la capacitación de los funcionarios públicos, los elegidos y los líderes comunitarios, la cual es proporcionada por entidades diversas (sector público, ONG, organizaciones internacionales, universidades y oficinas de consultores).

\subsection{Las localidades}

Tanto para entender mejor las nuevas formas de democracia representativa como para contextualizar la experiencia de democracia participativa aquí considerada, es importante enfocarse en los cambios que se impulsaron en las localidades. En efecto, en este ámbito territorial encontramos a la nueva JAL, la cual, no sin ambigüedades, tiene una doble misión: representativa y participativa.

En el distrito de Bogotá, las JAL constituyen una de las piezas fundamentales del programa del alcalde Castro, quien las creó en 1993 en aplicación de una ley de 1986 (Cf. Decreto 1421: Estatuto orgánico del distrito capital). Las funciones de la JAL son numerosas: El diseño, discusión y adopción del PDL, la gestión y el control de la ejecución de las inversiones del distrito en el ámbito de la localidad, la presentación de los proyectos de inversión, la aprobación del presupuesto anual, la promoción de la participación ciudadana en el control de los asuntos públicos y el control de la prestación de los servicios públicos. En cambio, los recursos con que cuentan el conjunto de las localidades por transferencia del distrito - si bien tienden a aumentar paulatinamente- son reducidos, pues sólo corresponden a cerca de $10 \%$ de los recursos del distrito, el cual es repartido entre las 20 localidades, dependiendo del tamaño poblacional de cada una de ellas.

La principal actividad de la JAL gira, entonces, alrededor de la elaboración del PDL y del seguimiento de su ejecución. Los proyectos que aparecen en estos planes consisten muy a menudo en construcciones o mejoramiento de infraestructuras (principalmente viales) y de edificios de función social (educación, salud, deportes y cultura, etc.) y poco en actividades de trabajo comunitario, desarrollo institucional o animación sociocultural. Esta preferencia puede ser ligada al hecho de que numerosos barrios tienen todavía necesidades importantes en estos campos. Pero proviene también del hecho de que los elegidos consideran ser juzgados por sus electores con base en resultados 'visualmente' identificables, 
tangibles, como lo permiten las obras de concreto (García y Zamudio, 1997).

La puesta en marcha de las JAL se efectuó mientras el tejido sociopolítico preexistente era fuertemente organizado entre el consejo distrital y las Juntas de Acción Comunal (JAC) (véase el cuadro 1), muchos concejales garantizaron su elección gracias a sus nexos con las JAC.

Desde la década de 1960, las JAC representan a los habitantes en el ámbito del barrio. Sus miembros son elegidos sólo por los habitantes del barrio que se afiliaron a ellas (el grado de afiliación puede ser muy variable de un barrio a otro) y su movilización depende mucho de la naturaleza de las necesidades del barrio. Es así como las JAC jugaron a menudo un papel determinante en los procesos de regularización de los barrios ilegales y de consecución de equipamiento en infraestructura y servicios públicos. En la década de 1990, la acción de estas organizaciones populares evolucionó, mientras que la movilización social y política en la sociedad en general se debilitó, las necesidades en servicios fueron paulatinamente satisfechas y se desarrollaron otras formas de organización. Sin embargo, en algunos sectores, las JAC siguen siendo bastante arraigadas y potentes.

Del otro lado, el consejo del distrito queda más estable, si bien la llegada de nuevas corrientes llamadas independientes favoreció la búsqueda de nuevos equilibrios. Esta estabilidad se debe en parte a su relación con las JAC.

Después de un arranque bastante confuso e improvisado, poco a poco las JAC definieron su espacio no tanto en paralelo, sino como intermediario entre el consejo y las JAC (Velásquez, 1996, García y Zamudio, 1997). Hoy en día, después de cuatro periodos (de 1993 a 2003), los tres ámbitos parecen estar articulados. Se ven cada vez más líderes de JAC que son elegidos ediles de JAL y luego concejales. Las redes sociopolíticas se adaptaron a esta nueva estructura político-administrativa.

Aunque con dificultades, dos grandes proyectos contribuyeron a que las JAL encontraran su legitimidad: la elaboración de los PDL y de los planes de desarrollo del distrito bajo los mandatos de los alcaldes Mockus y Peñalosa; enseguida, a partir de 1997, la elaboración del primer POT en la cual el CTP conformado por mitad de ediles de las JaL tenía que intervenir.

No obstante, las JAL y la administración local no han dejado de ser objeto de debates a veces muy polémicos: los mismos alcaldes distritales tienen opiniones divergentes sobre ellas. En nombre de 
la descentralización, Castro creó las JAL dotándolas de numerosas funciones, a pesar de que la coexistencia de algunas de ellas (la definición de proyectos en el PDL, la gestión y el control de su ejecución) pudo ser ambigua. Los alcaldes siguientes tuvieron una relación más compleja: Mockus las eludió creando a través de los encuentros ciudadanos espacios de concertación supuestamente más directos; de hecho, éstos fueron también controlados por las JAL. De manera más radical, Peñalosa les quitó sus competencias en materia de establecimiento de contratos para la ejecución de los proyectos del PDL y las transfirió a una nueva oficina del ámbito distrital (las Unidades Ejecutoras Locales, UEL), con el pretexto de que las JaL eran focos de corrupción.

Constatamos que la JAL encarna la tensión entre lo participativo y lo representativo: es percibida como una amenaza por el poder central; pero al mismo tiempo, la JAL supo insertarse rápidamente en el sistema sociopolítico, constituyendo un nuevo eslabón que facilitó las interacciones dentro de este mismo sistema. ¿Hasta qué punto es el conjunto de la ciudadanía la que se beneficia de sus gestiones?

\section{El control fiscal cívico: un procedimiento a priori innovador}

Como se ha señalado anteriormente, la participación consiste no sólo en la creación de instancias, sino también de procedimientos: es el caso del control fiscal cívico. Este programa promovido por la Contraloría de Bogotá tiene por misión controlar el buen manejo de los fondos públicos tanto en los ámbitos distrital y local. Se trata, entonces, de luchar contra la corrupción en las distintas etapas de la contratación, incluso cuando ésta se hace en el marco de las licitaciones supuestamente consideradas como transparentes, y contra cualquier anomalía en la ejecución de dichos contratos (la peor anomalía es el contrato pagado, aunque no ejecutado). Su principal papel es detectar estas fallas, pero no sancionar a los autores; por lo tanto esta institución actúa en complementariedad con las otras principales instituciones del derecho penal (la fiscalía y la procuraduría). Ella dispone de recursos bastante importantes, pero insuficientes, ya que debe examinar no sólo las ejecuciones de la administración distrital, sino también las de la administración local. Cabe recordar que el contralor es elegido por el consejo distrital.

En 1997 la Contraloría fue dirigida por un hombre que mantuvo una visión idealista de la misión de su institución, mientras 
que Mockus, el alcalde de ese entonces, ofrecía un discurso innovador de cultura ciudadana. En adhesión con los principios de la nueva Constitución, y como se promueve también en los ámbitos nacional y departamental, el contralor busca incorporar el control ciudadano de la ejecución de los fondos públicos en cada etapa de los procesos de la planificación y gestión distritales y locales.

El control fiscal consiste en la revisión de los contratos desde un punto de vista jurídico (según las reglas prescritas en la Ley 80 de 1993, cuyo objetivo es evitar cualquier clase de malversación de fondos), financiero (correspondencia entre las operaciones realizadas y las transacciones de fondos) y de gestión (cumplimiento de los proyectos previstos en los planes de desarrollo, eficacia en el desarrollo del contrato, etc.). El ejercicio del control fiscal cívico lleva a la consagración del derecho que tienen los ciudadanos de vigilar la cabal ejecución de los proyectos de financiamiento público, supuestos soportes del mejoramiento de su entorno cotidiano; la evaluación de esta cabal ejecución se basa en varios criterios, como los de economía, eficacia, eficiencia, equidad, costos ambientales. Los ciudadanos son considerados como los más motivados en exigir cumplimiento de parte de los contratistas y la administración pública local.

Por otra parte, un aspecto del programa de control fiscal cívico es muy importante para sus promotores: este control no puede ser correctamente ejercido por los ciudadanos si ellos no conocen claramente todo el proceso de planificación y gestión urbana. Es decir, se trata de propiciar una nueva cultura de los ciudadanos apoyada no tanto en la actitud de la denuncia (muy común en una sociedad en la cual los conflictos entre clanes políticos son a menudo canalizados por la justicia) sino en la responsabilidad (difícil cuando las prácticas de la planificación y gestión eran anteriormente mucho menos elaboradas y muy a menudo encerradas en las esferas administrativas).

Este programa se llevó a cabo durante varios años bajo el mandato de dos contralores, cada uno con un estilo propio, lo que incidió directamente en el manejo de este programa. Con el primer contralor, consistió en la selección -con base en las candidaturas presentadas en el marco de una licitación- de una organización cívica en cuatro localidades: Usaquén, Kennedy, Tunjuelito y Rafael Uribe (véase el mapa 1). Estas organizaciones eran contratadas y remuneradas por la contraloría para un año. Luego una capacitación específica: las organizaciones debían examinar alrededor de 70\% de los contratos establecidos en su localidad du- 


\section{Mapa 1 \\ División político-administrativa del distrito de Bogotá (1993)}

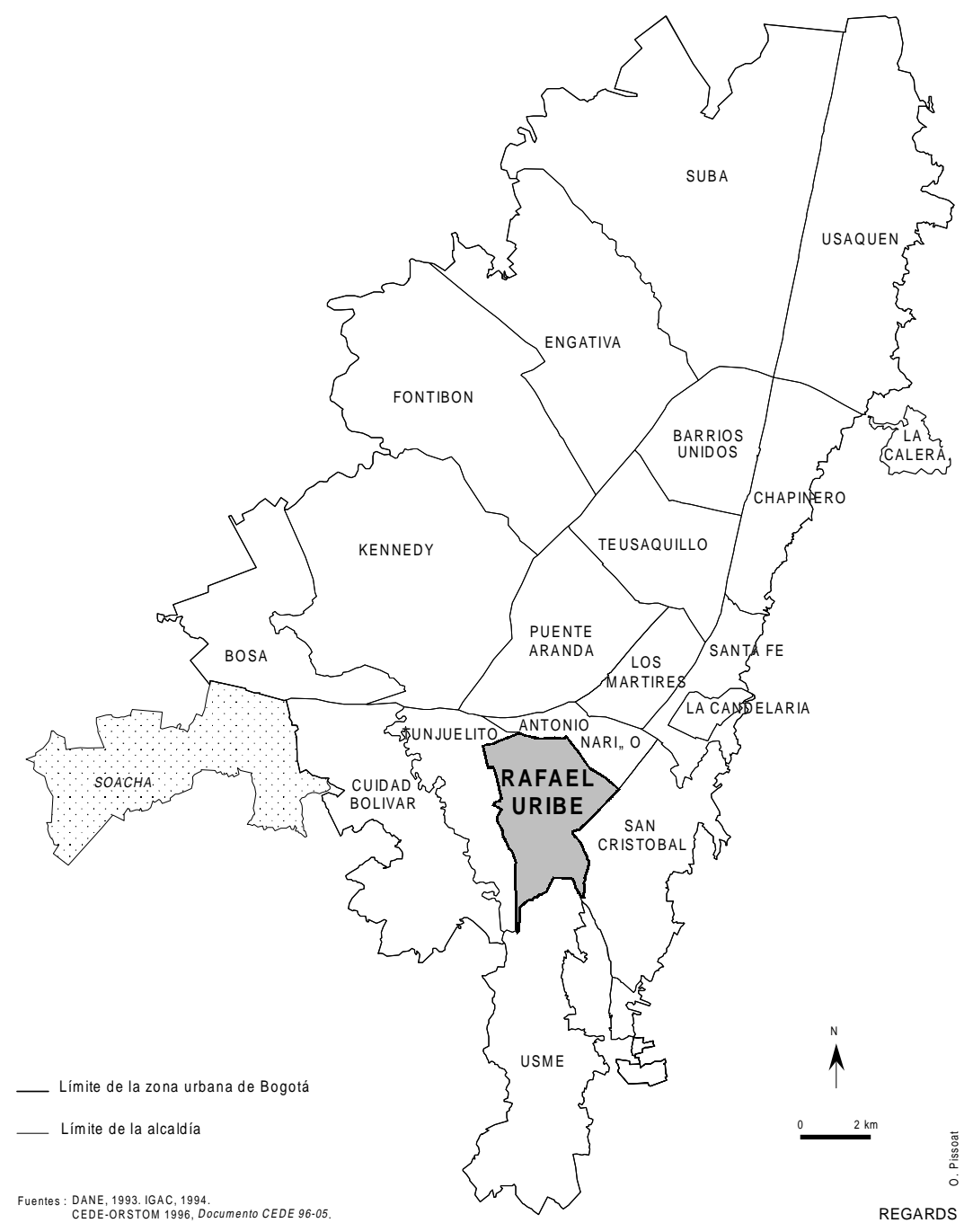


rante el año anterior dentro de la ejecución de los fondos locales y presentar los resultados de sus investigaciones en varios informes y en el marco de dos audiencias públicas (al final de cada).

Después de haber desarrollado el proceso en las tres primeras localidades, se dio un primer reajuste debido a varias dificultades: la complejidad de las labores, pero sobre todo el comportamiento conflictivo de la organización cívica de Usaquén frente a la alcaldía local. Aunque en los casos de Kennedy y Tunjuelito la situación no parece haber sido tan problemática, para el caso de Rafael Uribe se decidió recurrir a un equipo de universitarios (considerados como neutros y competentes), con el fin de que asumieran al tiempo el ejercicio del control fiscal y su transmisión progresiva a la población a través de sensibilización, información y capacitación. Estamos aquí en pleno debate sobre el poder del sabio frente al derecho del profano. Poco después de este reajuste tuvo lugar la elección del alcalde Peñalosa, y más tarde la de un nuevo contralor. Como se ha indicado, este alcalde cambió netamente de actitud frente a la administración de las localidades, privándolas de la competencia de contratación. Esta medida hizo caduco a partir de 1998 el ejercicio del control fiscal cívico en el ámbito local. El nuevo contralor, con aspiraciones mucho más pragmáticas (después de su mandato fue elegido representante en el Congreso), dejó finalizar el programa en las condiciones iniciales, pero luego se orientó hacia la capacitación masiva de todos los líderes comunitarios en torno a los nuevos procedimientos de la planificación y de la gestión (entre otros el del control fiscal, pero en detrimento de su práctica).

\section{La práctica del control fiscal cívico: relato de una experiencia compleja}

A continuación se presentará una parte de este programa, que fue realizada en la localidad de Rafael Uribe en 1998.

\subsection{Compromisos iniciales}

El contrato tenía dos objetivos: la realización del control fiscal en sí mismo y la sensibilización de la población con respecto de esta práctica. La intervención del equipo de universitarios consistía en las tareas siguientes a lo largo del año $1998:^{6}$

${ }^{6}$ Este equipo fue coordinado por el autor del presente artículo. 
- Una capacitación inicial en torno al plan de desarrollo, la ley de contratación, el presupuesto, la lectura y análisis jurídicos de los contratos;

- La definición de la muestra de los contratos a examinar (70\% del conjunto de los contratos ejecutados en el transcurso de 1997 por la administración de la localidad Rafael Uribe; es decir, 210 contratos) y la definición de herramientas de lectura y análisis de los contratos;

- El ejercicio directo del control fiscal con el análisis jurídico de los contratos (con el examen de 35 aspectos distintos de la contratación), así como en el caso de los contratos cuyo objeto era una obra de construcción (alrededor de $50 \%$ de los contratos), una visita técnica de la obra para evaluar el avance y calidad de su ejecución;

- La realización de dos audiencias a final de cada semestre con la presentación de los resultados de las investigaciones;

- El trabajo de sensibilización y capacitación de la población de la localidad en torno al control fiscal y a los procesos de planificación y gestión urbanas en curso, a partir de encuestas con los habitantes beneficiarios de los contratos evaluados.

El equipo reunía personas entre las cuales ninguna vivía en la localidad y cuyas formaciones y experiencias (especialmente en trabajo social e ingeniería civil) permitían cumplir con los compromisos. El apoyo jurídico debía provenir de la Contraloría.

\subsection{Rafael Uribe: una localidad con población diversa y una organización sociopolitica tradicional}

La localidad de Rafael Uribe se ubica en el pericentro sur de la ciudad (véase el mapa 1), alrededor de una colina, y reúne 125 barrios. Se urbanizó en su parte baja en la década de 1950 con la ocupación legal o no de tierras por numerosos migrantes (actualmente todo se encuentra legalizado), pero también con barrios planificados para clases medias hoy empobrecidas; la parte alta se urbanizó más tarde. La población de Rafael Uribe (cerca de 300 mil habitantes en 1997) pertenece en su mayoría (72.5\%) al estrato socioeconómico 3; es decir, a la clase media-baja. ${ }^{7}$ En ella se

\footnotetext{
${ }^{7}$ Bogotá está dividida en seis estratos socioeconómicos. La caracterización de los estratos se refiere a las características de las viviendas y el acceso a los servicios públicos domiciliarios. Se adoptó esta estratificación con el fin de definir las tarifas de consumo
} 
encuentran barrios ya consolidados, pero también barrios muy precarios que aparecieron recientemente en los últimos intersticios disponibles, es decir, a menudo en zonas de alto riesgo de desastre natural (deslizamientos, inundaciones, etc.); estos barrios son poblados sobre todo por personas víctimas de desplazamientos forzados o por hogares en situación de alta inestabilidad económica y social. Dependiendo de la historia del barrio (planificado, informal legalizado y consolidado, informal reciente), varía el grado de organización de la JAC - de fuerte a débil-y, por lo tanto, su papel actual en el desarrollo del barrio.

La política local, tal como se manifiesta en las elecciones de los once ediles de la JAL, es siempre muy marcada por el bipartidismo tradicional. Sin embargo, algunos independientes intentaron penetrar en el juego electoral. Las relaciones entre la JAL y los alcaldes locales a veces han sido muy tensas. Y las interacciones entre la JAL y los otros dos niveles de representación de la población (las JAC abajo, el consejo distrital arriba) son estrechas. Es un buen ejemplo de una JaL que vino a fortalecer y no obstaculizar, como se había podido pensar al principio, el juego clientelista (a través de la expresión de necesidades de la población y en el seguimiento de su satisfacción).

Los proyectos inscritos en el PDL de este periodo consistían en pavimentación o arreglos de vías, en construcción, ampliación o mantenimiento de colegios o centros de salud, en arreglos de parques, canchas de deportes, etc. El hecho de que el plan haya sido modificado dos veces después de su adopción oficial deja pensar que su elaboración se dificultó no sólo por la improvisación que reinó en ese entonces -acentuada por la exigencia de cuadrar los objetivos del plan local con la nueva formulación del plan del distrito-, sino también por diversas presiones políticas locales.

\subsection{Un procedimiento inacabado}

La realización del control fiscal cívico fue bastante compleja y demorada, tanto por su apropiación y ejecución como por una obstaculización inicial en el acceso a los contratos de parte de la administración local, la cual temía una intromisión demasiado grande.

de los servicios públicos, dependiendo de la capacidad de pago de los habitantes: muy bajas en los estratos inferiores ( 1 y 2 ) y muy altas en los estratos superiores (5 y 6 ). 
En estas condiciones, el trabajo de sensibilización de la población tuvo que ser bastante reducido: si bien se pudo divulgar el procedimiento de control fiscal a través de las encuestas de campo (212 personas encuestadas, alrededor de 50 obras), las audiencias públicas y un video, la transmisión colectiva y directa del método del control se habían vuelto imposibles.

Además, las dos audiencias públicas dominicales durante las cuales fueron presentados y discutidos los resultados y en las cuales intervinieron el contralor y sus funcionarios por un lado y la administración local por otro, reunieron a pocos participantes (alrededor de sesenta personas). Sin embargo, su perfil (ediles, líderes de JAC y otras organizaciones de las redes, pero muy pocos habitantes independientes) permitió a la JAL orientar hábilmente los debates: no se comentaron los resultados relativos a la gestión local, lo cual era el objetivo de estas audiencias, sino proyectos de la administración distrital. Ninguna de las fallas evidenciadas por el control fiscal pudo ser discutida públicamente.

\subsection{Resultados diversos}

Respecto del objetivo principal de lucha contra la corrupción, se detectaron pocos casos susceptibles de ser objeto de una encuesta más detallada: cerca de 20 contratos; es decir, 10\% del conjunto de los contratos revisados. Estos contratos consistían principalmente en obras inconclusas; en estos casos, los contratistas habían invocado que la duración demasiado larga de la tramitación del contrato había generado un desfase entre los costos reales y los inicialmente definidos; por lo tanto, decían tener dos opciones: realizar la obra hasta donde les permitía el presupuesto real, o hacerla toda pero con menor cantidad o calidad de materiales.

En cambio, la mayoría de los contratos habían sido establecidos y ejecutados de manera desordenada. Esta situación derivaba de una confusión general causada por la puesta en marcha de nuevos procedimientos, los de la planeación y gestión (el PDL fue modificado dos veces seguidas); pero provenía también del manejo mismo de los contratos: por ejemplo, falta de coincidencia entre el objeto del contrato y la necesidad a la cual pretendía responder, localización incorrecta, descriptivos y cotizaciones erróneas, informes de seguimiento incompletos, etc. Fue difícil analizar si, a la evidente pero comprensible falta de cultura técnica, no se sumaban también errores intencionales cometidos tanto por 
los contratistas como por la administración local, lo cual sí era sancionable.

\subsection{Obstáculos en la apropiación del procedimiento por los ciudadanos}

En cuanto a una posible apropiación de este tipo de procedimiento por la población, las entrevistas realizadas durante las visitas de campo permitieron identificar algunos obstáculos: las dificultades de la capacitación, la insuficiente motivación de la población no organizada, las condiciones confusas de elaboración del PDL.

Como se ha dicho, el procedimiento de control fiscal es fastidioso: no puede ser manejado sin una formación inicial sólida y sobre todo sin un estrecho acompañamiento por expertos. Se evidencia aquí una profunda contradicción, propia de este tipo de programa participativo: proviniendo de arriba, se exige que conceptos, métodos, lenguajes sabios sean reutilizados tal cual por la gente de abajo, es decir, profanos. Además, hace falta un trabajo pedagógico específico que pudiera tomar en cuenta la diversidad de los habitantes y las dinámicas sociales en las cuales están inmersos y, por tanto, las condiciones de su receptividad y apropiación de estos saberes. La población no es tan unificada como lo dejan suponer los discursos que preconizan la participación y el uso tan corriente de las nociones globalizantes de 'sociedad civil', 'ciudadanía', 'comunidad'.

El involucramiento de los habitantes no organizados en el control fiscal fue reducido. El bajo grado de su información sobre estos nuevos procedimientos y su íntima convicción de que la justicia colombiana no es eficaz, pudieron ser inhibidores, aunque su interés por evaluar obras era potencialmente alto. En cambio, en el caso de los habitantes organizados o con una formación o experiencias laborales adecuadas, se manifestó un mayor interés. Sin embargo, como se ha dicho, podían también ser presos de conflictos y alianzas entre actores locales que los movieron indujeron a apreciar el control fiscal no como un procedimiento que permitiera mejorar su entorno local, sino como un medio para denunciar a sus adversarios.

Las condiciones en las cuales se realizó el PDL determinaron directamente el interés por el control. Las visitas de campo y las entrevistas revelaron retrospectivamente cuáles habían podido ser las dinámicas de participación en la elaboración del PDL. Los proyectos inscritos en este plan no respondían siempre a las necesi- 
dades prioritarias. Era especialmente el caso de la pavimentación de vías que no accedían sino a unas pocas casas, a menudo una de ellas siendo ocupada por un miembro (o familiar de un miembro) de la JAC del barrio, mientras que la ampliación o remodelación de una escuela o un centro de salud utilizados por el conjunto de los habitantes eran esperados desde hace tiempo. El papel de estos juegos clientelistas (con una red que asocia líderes de JAC, ediles de JAL, concejales del distrito) queda, por lo tanto, predominante y sesga la puesta en marcha de las reglas de una nueva planeación racional y equitativa. Al percibir esta profunda contradicción, hemos sido llevados a leer esta experiencia desde otra perspectiva.

\section{5. ¿A quién le sirvió el control fiscal cívico?}

Resulta de gran interés reubicar este programa en un sistema de actores que lo perciben como una ganancia o una amenaza y, por consiguiente, se organizan, se alían o se oponen entre sí. Este sistema está constituido por tres actores principales: ${ }^{8}$

- La jal y la administración local (el alcalde local y su administración).

- Los habitantes, en especial los organizados a través de las JAC.

- La contraloría.

En la lógica del control fiscal cívico, el primer actor principal es sospechoso, el segundo es la víctima, y el tercero es el justiciero. De hecho, la lógica parece haber sido otra.

Pensamos que el proceso tal como se desarrolló en Rafael Uribe contribuyó a la reorganización de un sistema sociopolítico basado en el modelo tradicional del clientelismo. En este sistema se encuentran implicados la mayoría de los elegidos (en todos los ámbitos territoriales), pero también los electores mismos; y sigue todavía activo en otros términos, mientras que el objetivo principal del control fiscal cívico era precisamente luchar contra las prácticas clientelistas, suponiendo que éstas van en contra de los intereses de los habitantes, el resultado sería, por el contrario, un fortalecimiento de un sistema en el cual los habitantes organizados parecen encontrar satisfacción. No se podía esperar que los beneficiarios del clientelismo denunciaran el funcionamiento del mismo. Es así como el espacio del joven pero frágil poder partici- 
pativo parece haber sido ocupado, incluso fagocitado, por el usado pero hábil poder representativo. La relectura del comportamiento de los tres principales actores involucrados en esta experiencia permite plantear mejor nuestra hipótesis.

\subsection{Una administración local solidaria}

La JAL de Rafael Uribe es atravesada por las tendencias políticas tradicionales y muy marginalmente por las nuevas independientes. Pero frente al control existió entre los 11 ediles un relativo consenso de defensa. En efecto, para los ediles, el control constituía una amenaza, pues hacía percibir como sospechosas sus prácticas en la definición de los contratos. Esta amenaza los llevó a movilizar un conjunto de fuerzas sociopolíticas y, al mismo tiempo, dar otro papel a su corporación: ya no la gestión del desarrollo, sino la (re)activación de la mecánica representativa.

Esta actitud de defensa por parte de los ediles se manifestó en dos puestas en escena distintas: en el recinto mismo de la JAL; es decir, en su propio territorio, con reacciones unánimes frente a preguntas sobre su papel en los nuevos procesos de gestión, rechazando cualquier sospecha sobre sus posibles actuaciones indebidas en la gestión de los contratos; en las audiciones públicas, durante las cuales de forma estratégica algunos ediles dirigían varias quejas a la contraloría, pero muy generales, y enseguida hacían expresar - por sus 'socios' líderes de las JAC -9 fuertes dudas sobre la real viabilidad y eficacia del programa de control fiscal cívico.

\subsection{Unos habitantes cómplices}

En la población, importa diferenciar al menos dos tipos de habitantes: los primeros están ligados a organizaciones sociales, principalmente las JAC, los segundos están completamente fuera de estos procesos cívicos; estos últimos reflejan la situación de su propio grupo social, fuertemente marcado por la precariedad, e inclusive la exclusión social. Ellos son los primeros que nos interesan.

Como se ha señalado, estos habitantes tenían nexos directos con los ediles, lo que fue determinante desde la elaboración del plan local. En las audiencias, su actitud llevó a una cierta negación de lo que se pretendía hacer con el control fiscal cívico: en el debate hicieron pocas referencias a los contratos realizados en los barrios bajo la responsabilidad de la alcaldía local; parecía impor- 
tarles más los proyectos mal manejados por la administración distrital. Este desfase entre el objeto de las audiencias -el control de la ejecución de las inversiones en el ámbito local- y la naturaleza de las críticas en ellas formuladas -la no planificación y ejecución de inversiones del distrito-, no se interpreta fácilmente. Obviamente los proyectos financiados por el distrito también tienen que ver directamente con la vida cotidiana de los barrios; sin embargo, no era el ámbito para expresar estas insatisfacciones. Este error podía provenir de un desconocimiento de las reglas del juego, las cuales se vuelven cada vez más complejas. Ahora bien, como lo observamos anteriormente en el caso de los ediles, estas intervenciones pudieron derivar también de una estrategia de protección del funcionamiento clientelista local del cual estos mismos grupos son directamente beneficiarios.

\subsection{Una contraloría ambigua}

A pesar de la naturaleza de su misión, la contraloría se inscribe también en el juego político, puesto que el contralor es elegido por el consejo del distrito. Por lo tanto, sus programas pueden ser utilizados con otros fines que las enunciadas oficialmente. Es importante recontextualizar aquí la acción de cada contralor involucrado en el programa. La del primero se inscribía en la política municipal de Mockus enfocada hacia la 'cultura ciudadana', es decir, la formación de un ciudadano 'responsable', comprometido con su localidad independientemente de las corrientes políticas tradicionales y de los juegos clientelistas. Sin embargo, se mostró bastante ingenua al creer posible la puesta en práctica de un programa muy complejo.

La del segundo se relaciona con tensiones entre el distrito y las localidades. Desde el principio de su mandato, el alcalde Peñalosa privó a las JAL de sus competencias en materia de contratación para atribuírselas a su propia administración. También facilitó el regreso a un cierto régimen de favores en la atribución de las grandes obras del distrito y al clientelismo en el ámbito del consejo. En este último contexto, la orientación que tomó el segundo contralor es bastante coherente puesto que se trata, a través de la formación masiva de los líderes de JAC, de reactivar la relación directa distrito-barrio, útil en la perspectiva de aspiraciones políticas personales. 


\section{Conclusión}

En primer lugar, se confirma que la práctica de la participación es más compleja que lo enunciado en los discursos sobre el tema. En segundo lugar, este programa resultó ser excluyente: aunque se ejerció el control fiscal-concebido inicialmente no sólo como un procedimiento jurídico, sino también como una evaluación del papel de la planeación y gestión locales en el mejoramiento de los espacios públicos y servicios sociales de las localidades- no fue fácilmente apropiado por los ciudadanos. Además, los habitantes más necesitados, a menudo los menos organizados, accedieron poco a estos procedimientos y se beneficiaron poco de este mejoramiento. En cambio, el sistema sociopolítico municipal y local, a través de la JAL y las JAC, supo utilizar este programa para consolidar el funcionamiento de sus redes, aprovechándose hábilmente de los nuevos espacios así propiciados. Superó el riesgo de ser fiscalizado, dado que sus prácticas tradicionales, a menudo marcadas por el clientelismo, no se prestaban a una gestión racional, transparente e incluyente.

Esta conclusión sacada del análisis de una experiencia específica, pero atravesada por problemáticas muy generales, nos invita a reflexionar no tanto sobre la planeación y la gestión participativas y sus mecanismos, sino el lugar de la participación dentro de los nuevos procedimientos y espacios políticos promovidos a través de la descentralización y la democratización. No se puede disociar la participación ciudadana de la representación política. El componente participativo es objeto de mucha idealización en los discursos oficiales, lo que tiene varios efectos: no puede ser evaluado de forma objetiva, su percepción como amenaza por algunos responsables políticos no puede ser explícitamente señalada, opaca los intentos de renovación de la representación política acorde con los cambios sociales y culturales vividos por los ciudadanos, incluso puede ser utilizado para mantener el carácter tradicional de la representación política. En otros términos, ni el primer componente se renueva ni el segundo se desarrolla; la democratización no puede ser tan efectiva como se había deseado, y por lo tanto la planeación y gestión participativas no se consolidan. La fragilidad de las reformas frente a la fuerza de los tejidos sociopolíticos podría amenazar el desarrollo de nuevas formas de interacciones entre planificadores y habitantes. 


\section{Bibliografía}

Atkinson, R. (1998), "Les aléas de la participation des habitants à la gouvernance urbaine en Europe", Les annales de la recherche urbaine, núm. 80-81, Paris, pp. 75-83.

Blanc, M. (1995), "Politique de la ville et démocratie locale", Les annales de la recherche urbaine, núm. 68-69, Paris, pp. 99-106.

CNUAH (1996), Un mundo en proceso de urbanización, informe mundial sobre los asentamientos humanos, tomo 2, TM editores, Inurbe, FNA, Bogotá.

García, M.C. y J. V. Zamudio (1997), Descentralización en Bogotá bajo la lupa (1992-1996), CINEP, Bogotá.

Gouëset, V. (1998), Bogotá, nacimiento de una metrópoli. La originalidad del proceso de concentración urbana en Colombia en el siglo XX, TM editores, Bogotá.

Lorcerie, F. (1995), "L'université du citoyen à Marseille", Les annales de la recherche urbaine, núm. 68-69, París, pp.123-134.

Pécaut, D. (1987), L'ordre et la violence. Evolution socio-politique de la Colombie entre 1930 et 1953, Editions de L'EHESs, París.

Velásquez, F. (1996), Ciudad y participación, Editorial Universidad del Valle, Cali.

Velásquez, F. y E. González (2003), ¿Qué ha pasado con la participación ciudadana en Colombia?, Fundación Corona, Fundación Social, Foro Nacional por Colombia, Banco Mundial, CIDER-Universidad de Los Andes, Corporación Región, Viva la Ciudadanía, Transparencia por Colombia, Bogotá. 
Vidal, D. (1998). La politique au quartier. Rapports sociaux et citoyenneté à Recife, Ed. MSH, París.

Enviado: 21 de junio de 2003. Aceptado: 15 de diciembre de 2003. 